\title{
Role for Salicylic Acid in the Activation of Defense Responses in Catalase-Deficient Transgenic Tobacco
}

\author{
He Du and Daniel F. Klessig \\ Waksman Institute and Department of Molecular Biology and Biochemistry, Rutgers, the State University of \\ New Jersey, P.O. Box 759, Piscataway 08855, U.S.A. \\ Received 19 March 1997. Accepted 5 June 1997.
}

\begin{abstract}
Transgenic tobacco plants with severely reduced catalase activity were crossed with NahG plants that do not accumulate salicylic acid. Some of the progeny from the cross spontaneously developed necrosis when grown under strong light, similar to that observed on the parental severely catalase-deficient plants. However, in contrast to the parental catalase-deficient plants, these progeny plants did not constitutively express $P R-1$ genes or develop enhanced resistance. Thus, salicylic acid appears to be required for the induction of these defense responses in catalase-deficient tobacco plants.
\end{abstract}

In tobacco (Nicotiana tabacum), as well as in many other plants, pathogen infection of resistant cultivars results in the hypersensitive response (HR), which is characterized by the formation of necrotic lesions at the site of infection and the restriction of pathogen growth and spread. In addition to this local resistance response, tissues distal to the infection site develop enhanced resistance to a secondary infection by the same or even unrelated pathogens. This enhanced disease resistance is generally termed systemic acquired resistance (SAR; for review, see Ryals et al. 1996; Wobbe and Klessig 1996). Associated with the HR and SAR is the expression of defense-related genes, such as those encoding the pathogenesis-related (PR) proteins. Synthesis of PR proteins has been widely used as a molecular indicator for the activation of plant defense mechanisms.

Salicylic acid (SA) has been implicated as one of the key components in the signal transduction pathway leading to plant resistance to various pathogens (Ryals et al. 1996; Wobbe and Klessig 1996). For instance, in tobacco, endogenous SA levels increase after pathogen attacks, which correlates with the expression of $P R$ genes and the onset of SAR. Secondly, exogenous application of SA also induces $P R$ gene expression and enhanced disease resistance. Thirdly, transgenic tobacco plants constitutively expressing the bacterial $n a h G$ gene (which encodes salicylate hydroxylase, an enzyme that destroys SA) show enhanced susceptibility to pathogen attacks and fail to develop SAR (Gaffney et al. 1993; Delaney et al. 1994).

Corresponding author: Daniel F. Klessig; Telephone: 732-445-3805; Fax: 732-445-5735; E-mail: klessig@ mbcl.rutgers.edu
In an attempt to elucidate the mechanisms of SA action in plant disease resistance, we have identified an SA-binding protein in tobacco leaves (Chen and Klessig 1991; Chen et al. 1993a, 1993b). It is catalase. The discovery that SA inhibited catalase's $\mathrm{H}_{2} \mathrm{O}_{2}$-degrading activity and that prooxidants such as $\mathrm{H}_{2} \mathrm{O}_{2}$ induced $P R$ gene expression led to the proposal that one mechanism of SA's action is elevation of the level of $\mathrm{H}_{2} \mathrm{O}_{2}$ or $\mathrm{H}_{2} \mathrm{O}_{2}$-derived reactive oxygen species (ROS), which then serve as intermediates in the SA signal transduction pathway (Chen et al. 1993b). Consistent with this model, the following were observed: (i) 2,6-dichloroisonicotinic acid (INA) and benzothiadiazole (BTH), two synthetic inducers of $P R$ genes and enhanced resistance, also inhibit tobacco catalase (Conrath et al. 1995; Wendehenne et al., in press); (ii) SA, INA, and BTH inhibit the activity of tobacco ascorbate peroxidase, the other key $\mathrm{H}_{2} \mathrm{O}_{2}$-scavenging enzyme, in vitro (Durner and Klessig 1995; Wendehenne et al., in press); and (iii) antioxidants suppress SA-, INA-, or BTH-induced $P R-1$ gene expression (Conrath et al. 1995; Wendehenne et al., in press).

The involvement of catalase inhibition by SA and elevated $\mathrm{H}_{2} \mathrm{O}_{2}$ levels in plant defense responses, however, has been called into question based on four observations: (i) tobacco plants infected with Pseudomonas syringae or leaf disks treated with SA did not show significant reduction in catalase activity (Bi et al. 1995); (ii) $\mathrm{H}_{2} \mathrm{O}_{2}$ levels did not increase during the establishment of SAR (Neuenschwander et al. 1995); (iii) $P R-1$ gene activation by prooxidants such as $\mathrm{H}_{2} \mathrm{O}_{2}$ or 3aminotriazole was suppressed in NahG transgenic tobacco (Bi et al. 1995; Neuenschwander et al. 1995); and (iv) very high levels of $\mathrm{H}_{2} \mathrm{O}_{2}$ induced the production of SA (León et al. 1995; Neuenschwander et al. 1995; Summermatter et al. 1995). These results argue that $\mathrm{H}_{2} \mathrm{O}_{2}$ acts upstream of $\mathrm{SA}$ in the signal transduction pathway rather than, or in addition to, acting downstream of SA.

To further investigate the involvement of catalase and ROS in the activation of defense responses, transgenic tobacco plants with severely depressed levels of catalase due to either sense co-suppression or antisense suppression were constructed (Chamnongpol et al. 1996; Takahashi et al. 1997). These plants spontaneously develop necrosis under high light, accumulate PR proteins, and show enhanced disease resistance. However, the antisense-suppressed, catalase-deficient plants (ASCAT1; Takahashi et al. 1997) also exhibited slightly 
to moderately elevated levels of SA and its glucoside. Thus, it is unclear whether the activation of defense responses was due to the reduction in catalase activity or the increase in SA levels.

To resolve this question, we have crossed two plants from the ASCAT1 \#17 line (T2 generation; Takahashi et al. 1997) with homozygous NahG-10 plants (Gaffney et al. 1993). A total of $10 \mathrm{~F} 1$ progeny from two crosses (AN1 \#1 to \#5 and AN2 \#1 to \#5; Fig. 1A) were analyzed. Since the parental ASCAT1 \#17 transgenic plant contained multiple copies of the antisense catalase insert (data not shown) and one or several of the insertions may be in a heterozygous state in the T2 plants, the catalase-deficient phenotype was still segregating. The two T2 plants of ASCAT1 \#17 were also crossed with wild-type (wt) tobacco plants; progeny of these crosses (AW1 $\# 1$ to \#5 and AW2 \#1 to \#5; Fig. 1A) served as controls to
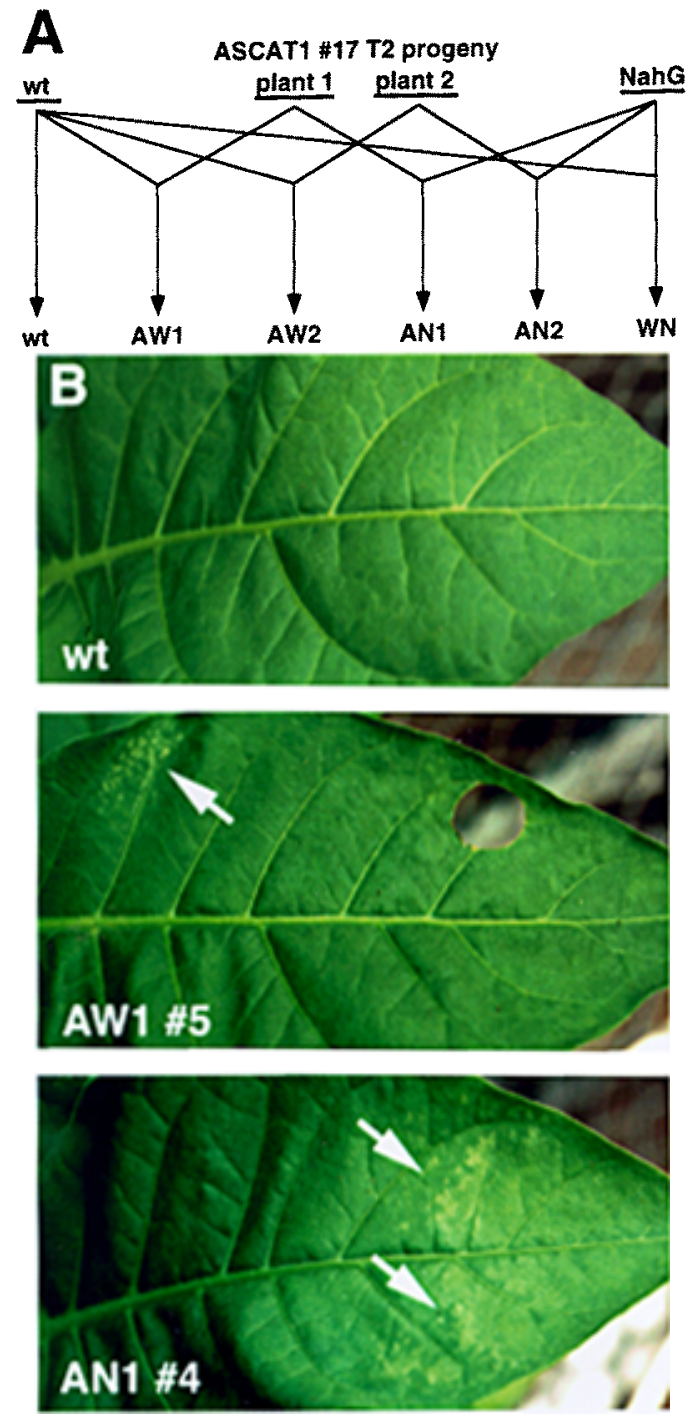

Fig. 1. A, Schematic representation of crosses performed between wildtype (wt), severely catalase-deficient tobacco (ASCAT1 \#17 T2 generation), and NahG-10 plants. Abbreviations representing the progeny from each cross are listed at the bottom. B, Photographs of a wt leaf and the leaves of progeny from two crosses showing areas of spontaneously developing necrosis (indicated by arrows). which the AN1 and AN2 plants were compared. As the AN1 and AN2 plants were heterozygous for the $n a h G$ gene, progeny of a cross between the NahG plant and the wt plant served as another control (WN; Fig. 1A). All plants were grown at $20^{\circ} \mathrm{C}$ under a 14-h light cycle with strong light $(20,000$ to 28,000 lx).

Similar to the parental ASCAT1 plants, lesions spontaneously developed on the leaves of five AW and AN plants (Fig. 1B). In contrast, none of the six wt and $\mathrm{WN}$ plants developed lesions under these conditions. Due to the presence of multiple copies of the antisense catalase insert, AW and AN plants exhibited heterogeneity with respect to catalase protein levels and catalase activity (Figs. 2 and 3). Interestingly, the five plants (AW1 \#2, AW1 \#5, AN1 \#2, AN1 \#4, and AN2 \#3) that had the most severely reduced catalase activity (Fig. 3) and the lowest levels of catalase protein (Fig. 2), also spontaneously developed necrosis (Fig. 2), while the others did not. This is consistent with the previous finding that development of necrosis correlated with greatly reduced catalase activity (>90\%; Takahashi et al. 1997). Since these AN plants with severely depressed catalase levels developed necrosis just like

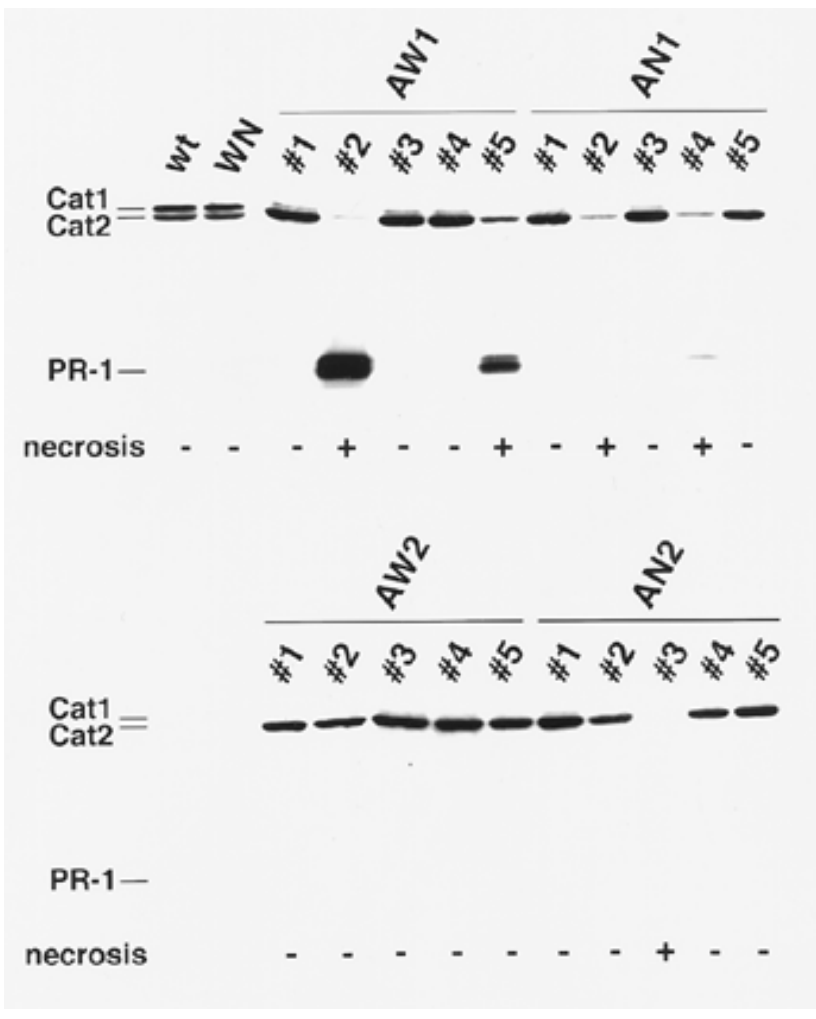

Fig. 2. Western blot (immunoblot) analysis of catalases and PR-1 proteins in leaf extracts. Leaf disks were taken either from leaves showing necrosis or from leaves at similar developmental stage on other phenotypically-normal plants. Soluble leaf proteins were extracted in buffer containing $50 \mathrm{mM}$ Tris- $\mathrm{HCl} \mathrm{pH} 7.5,1 \mathrm{mM}$ EDTA, $10 \mathrm{mM} \beta$-mercaptoethanol, and $10 \mu \mathrm{g}$ of phenylmethylsulfonyl fluoride per ml. Protein (10 $\mu \mathrm{g}$ per lane) was separated on a $12 \%$ sodium dodecyl sulfate-polyacrylamide gel and then transferred onto a nitrocellulose membrane. Monoclonal antibodies (MAb3B6, MAb1F5, and MAb33G1) were used to detect catalase 1 (Cat1), catalase 2 (Cat2), and PR-1 proteins. Presence $(+)$ or absence $(-)$ of spontaneously developing necrosis is indicated at bottom of each lane. The number corresponds to individual progeny from the various crosses. See Figure 1A and text for description of the different crosses. 


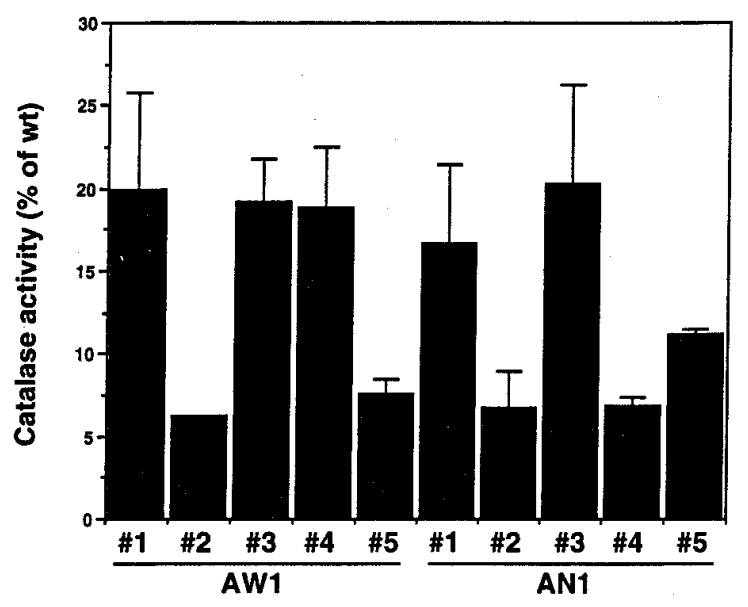

Fig. 3. Catalase activity present in the leaf extract. Catalase activity was measured with an $\mathrm{O}_{2}$ evolution assay according to Takahashi et al. (1997). Catalase activity of the wild-type (wt) plants was defined as $100 \%$. WN plants contain levels of catalase activity similar to those of the wt plants (data not shown). Catalase activity and standard deviation (shown as an error bar) were calculated for each plant with three independent extracts from the same leaf.

the severely catalase-deficient AW plants, elevated levels of SA do not appear to be required for the formation of necrosis. In contrast, SA seems to be required for the accumulation of PR-1 proteins in these catalase-deficient plants (Fig. 2). While the AW1 \#2 and AW1 \#5 plants accumulated substantial amounts of PR-1 proteins, AN1 \#2, AN1 \#4, and AN2 \#3 plants produced little, if any, PR-1 proteins, even though they had very low levels of catalase and spontaneously developed necrosis. Thus, while stress resulting from severely reduced catalase activity likely induced necrosis, it appears to be insufficient for the activation of certain defense responses in the absence of elevated levels of SA.

SA was also necessary for the induction of enhanced resistance (i.e., SAR) to tobacco mosaic virus (TMV) in the catalase-deficient plants. As anticipated from our previous study (Takahashi et al. 1997), the severely catalase-deficient plants AW1 \#2 and AW1 \#5 not only developed necrosis and constitutively expressed $P R-1$ genes, but also formed smaller lesions after TMV infection than the wt control or the other AW progeny with less depressed catalase levels (Fig. 4). In marked contrast, the two severely catalase-deficient AN progeny (AN1 \#2 and AN1 \#4) while developing necrosis, not only failed to express $P R-1$ genes, but also formed TMV-induced lesions indistinguishable in size from those on the WN control and other AN plants with less depressed catalase levels.

In summary, our results indicate that $\mathrm{SA}$ is required for the activation of certain defense responses, such as $P R-1$ gene expression and the development of enhanced resistance in the catalase-deficient progeny derived from crosses between ASCAT1 \#17 and the NahG plants. However, SA does not appear to be required for the development of necrosis, which is likely brought about by stress resulting from the severe reduction of catalase activity. This latter conclusion is consistent with the results from several other studies, which indicate that necrotic lesion formation can be uncoupled from disease resistance. For example, when NahG transgenic Arabidopsis plants were crossed to the lesion-forming Arabidopsis mutants $l s d 2$ and

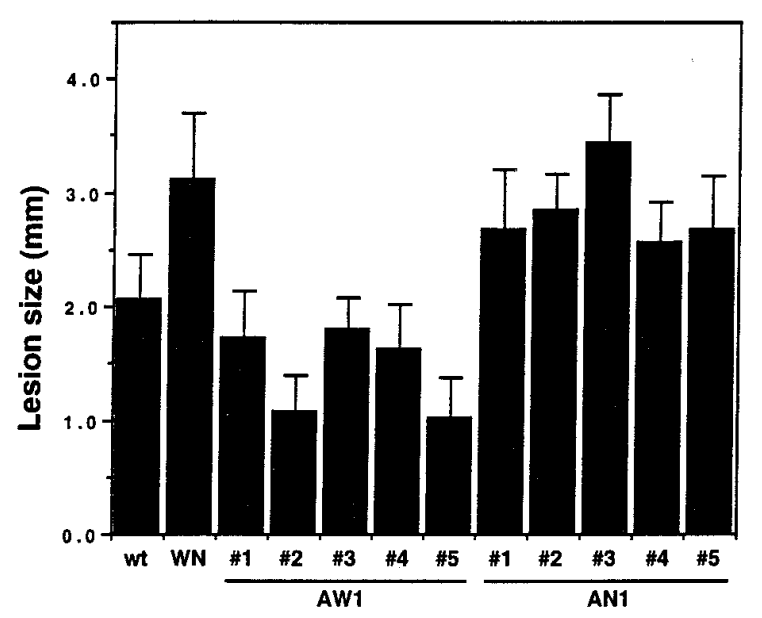

Fig. 4. Lesion size 7 days post tobacco mosaic virus (TMV) infection. Leaves were inoculated with TMV according to Takahashi et al. (1997). Diameters of 20 randomly picked lesions from the same leaf were measured and the standard deviation (shown as an error bar) was calculated.

$l s d 4$ (Dietrich et al. 1994), the progeny did not express $P R-1$ genes or show enhanced disease resistance; lesion formation, however, was unaffected (Ryals et al. 1996). Another Arabidopsis mutant, $n d r l$, is susceptible to several pathogens that are avirulent on wt Arabidopsis. Interestingly, an HR similar to that occurring in wt plants also develops on ndrl mutant upon pathogen infection (Century et al. 1995). Finally, while NahG tobacco plants show enhanced susceptibility to several elicitin-producing Phytophthora spp. and fail to establish SAR after elicitin treatment, the necrosis response is unchanged with respect to that observed in wt tobacco plants (Keller et al. 1996). Hence, there seems to be an SA-independent pathway controlling the formation of necrosis, in addition to the SAdependent pathway that leads to the induction of $P R$ genes and enhanced resistance.

\section{ACKNOWLEDGMENTS}

We wish to thank all members of the Klessig laboratory, in particular Jyoti Shah and D'Maris Dempsey for helpful discussion and critical reading of the manuscript. We also thank John Ryals for generously providing seeds from the NahG-10 transgenic tobacco line. This work was supported in part by grant MCB-9514239 from the National Science Foundation.

\section{LITERATURE CITED}

Bi, Y. M., Kenton, P., Mur, L., Darby, R., and Draper, J. 1995. Hydrogen peroxide does not function downstream of salicylic acid in the induction of PR protein expression. Plant J. 8:235-245.

Century, K. S., Holub, E. B., and Staskawicz, B. J. 1995. NDR1, a locus of Arabidopsis thaliana that is required for disease resistance to both a bacterial and a fungal pathogen. Proc. Natl. Acad. Sci. USA 92: 6597-6601.

Chamnongpol, S., Willekens, H., Langebartels, C., Van Montagu, M., Inzé, D., and Van Camp, W. 1996. Transgenic tobacco with a reduced catalase activity develops necrotic lesions and induces pathogenesisrelated expression under high light. Plant J. 10:491-503.

Chen, Z., and Klessig, D. F. 1991. Identification of a soluble salicylic acid-binding protein that may function in signal transduction in the plant disease resistance response. Proc. Natl. Acad. Sci. USA 88: 8179-8183.

Chen, Z., Ricigliano, J. W., and Klessig, D. F. 1993a. Purification and 
characterization of a soluble salicylic acid-binding protein from tobacco. Proc. Natl. Acad. Sci. USA 90:9533-9537.

Chen, Z., Silva, H., and Klessig, D. F. 1993b. Active oxygen species in the induction of plant systemic acquired resistance by salicylic acid. Science 262:1883-1886.

Conrath, U., Chen, Z., Ricigliano, J. W., and Klessig, D. F. 1995. Two inducers of plant defense responses, 2,6-dichloroisonicotinic acid and salicylic acid, inhibit catalase activity in tobacco. Proc. Natl. Acad. Sci. USA 92:7143-7147.

Delaney, T. P., Uknes, S., Vernooij, B., Friedrich, L., Weymann, K., Negrotto, D., Gaffney, T., Gut-Rella, M., Kessmann, H., Ward, E., and Ryals, J. 1994. A central role of salicylic acid in plant disease resistance. Science 266:1247-1250.

Dietrich, R. A., Delaney, T. P., Uknes, S. J., Ward, E. R., Ryals, J. A., and Dangl, J. L. 1994. Arabidopsis mutants simulating disease resistance response. Cell 77:565-577.

Durner, J., and Klessig, D. F. 1995. Inhibition of ascorbate peroxidase by salicylic acid and 2,6-dichloroisonicotinic acid, two inducers of plant defense responses. Proc. Natl. Acad. Sci. USA 92:11312-11316.

Gaffney, T., Friedrich, L., Vernooij, B., Negrotto, D., Nye, G., Uknes, S., Ward, E., Kessmann, H., and Ryals, J. 1993. Requirement of salicylic acid for the induction of systemic acquired resistance. Science 261: 754-756.

Keller, H., Bonnet, P., Galiana, E., Pruvot, L., Friedrich, L., Ryals, J., and Ricci, P. 1996. Salicylic acid mediates elicitin-induced systemic acquired resistance, but not necrosis in tobacco. Mol. Plant-Microbe Interact. 9:696-703.

León, J., Lawton, M. A., and Raskin, I. 1995. Hydrogen peroxide stimulates salicylic acid biosynthesis in tobacco. Plant Physiol. 108:16731678.

Neuenschwander, U., Vernooij, B., Friedrich, L., Uknes, S., Kessmann, H., and Ryals, J. 1995. Is hydrogen peroxide a second messenger of salicylic acid in systemic acquired resistance? Plant J. 8:227-233.

Ryals, J. A., Neuenschwander, U. H., Willits, M. G., Molina, A., Steiner, H.-Y., and Hunt, M. D. 1996. Systemic acquired resistance. Plant Cell 8:1809-1819.

Summermatter, K., Sticher, L., and Métraux, J.-P. 1995. Systemic responses in Arabidopsis thaliana infected and challenged with Pseudomonas syringae pv. syringae. Plant Physiol. 108:1379-1385.

Takahashi, H., Chen, Z., Du, H., Liu, Y., and Klessig, D. F. 1997. Development of necrosis and activation of disease resistance in transgenic tobacco plants with severely reduced catalase levels. Plant J. 11:9931005.

Wendehenne, D., Durner, J., Chen, Z., and Klessig, D. F. Benzothiadiazole, an inducer of plant defenses, inhibits catalase and ascorbate peroxidase. Phytochemistry. (In press.)

Wobbe, K. K., and Klessig, D. F. 1996. Salicylic acid - an important signal in plants. Pages 167-196 in: Plant Gene Research Series, Signal Transduction in Plant Growth and Development. D. P. S. Verma, ed. Springer-Verlag, Vienna. 